\title{
Gravity, inertia and dark energy
}

\section{Peter Rowlands}

Physics Department, University of Liverpool, Oliver Lodge Laboratory, Oxford St, Liverpool, L79 7ZE, UK

\section{Email address:}

p.rowlands@liverpool.ac.uk

\section{To cite this article:}

Peter Rowlands. Gravity, Inertia and Dark Energy. American Journal of Modern Physics. Special Issue: New Science Light Path on Cosmological Dark Matters. Vol. 4, No. 1-1, 2015, pp. 18-22. doi: 10.11648/j.ajmp.s.2015040101.14

\begin{abstract}
Measurements of the density parameter of the universe $\Omega$ have slowly converged towards the critical value of 1, where the universe is defined to be flat and Euclidean. New data provided by the Planck probe suggest there may be a critical value for the dark energy component of this parameter $\Omega_{\Lambda}$. A physical significance may occur at $\Omega_{\Lambda}=2 / 3$ and be connected with the origin of inertia. If future observations constrain $\Omega_{\Lambda}$ to exactly this value, the implication is that dark energy originates in constraints provided by fundamental laws of physics on possible cosmologies for the universe.
\end{abstract}

Keywords: Dark Energy, Gravitation, Inertia, Mach’s Principle, Cosmological Constant

\section{Introduction}

Dark energy, as usually presented, was an entirely unexpected phenomenon, first appearing in 1998 as an acceleration term in the velocities for type Ia supernovae in distant galaxies $[1,2]$. Though it was soon linked with the cosmological constant, originally introduced by Einstein to prevent the universe from collapsing in on itself under its own gravity, its effect was completely different, leading to increased expansion rather than preventing contraction. Theories of quantum gravity seemed to suggest that a vacuum effect could produce an outward pressure equivalent to that of the cosmological constant. However, estimates of the value for the density led to what is probably the worst ever match between theory and experiment in the history of physics.

Essentially, if we assume one particle with the Planck mass in every Compton volume or Compton radius cubed, then the vacuum density will be of order $m_{P}^{4} c^{3} / \hbar^{3}=(\hbar c / G)^{2} c^{3} / \hbar^{3}=$ $c^{5} / \hbar G^{2}=5.16 \times 10^{96} \mathrm{~kg} \cdot \mathrm{m}^{-3}$. However, the experimental evidence from the supernova measurements provides a measured vacuum density of order $0.7 \times 10^{-26} \mathrm{~kg} \cdot \mathrm{m}^{-3}$, meaning our estimates are incorrect by a factor of $7.37 \times 10^{122}$. Even using the non-reduced Compton radius and applying geometric factors would not reduce this factor by more than two or three orders of magnitude. The only possible conclusion from a result so badly wrong is that 'quantum gravity' as it is normally conceived cannot be the origin of the dark energy. In such cases we have to begin with the experimental result rather than the most-favored theoretical projection, and it turns out that this is indeed very suggestive of the real meaning of dark energy. First we need to look at how the cosmological constant relates to gravity in the conventional theory, and, because of how the universe appears to be structured, it makes no difference whether we use general relativity or Newtonian gravity. Nor, despite the statements found in many written accounts, does it make any significant difference whether or not we tie the result to any particular theory of cosmology.

\section{The Cosmological Constant}

We begin by using general relativity and the possible cosmologies that emerge from the Friedmann solutions of the Einstein field equations [3]. For an expanding universe, the Hubble parameter $H$ is defined as the normalized rate of expansion $H=\dot{R} / R$, and is measured as the ratio $v / r$ of the recessional velocity $v$ and the co-moving distance $r$ of distant galaxies, where $R$ is an assumed scale factor. The Hubble parameter at the present time, which is known as the Hubble constant $H_{0}$, can be defined in terms of the Hubble radius $r_{H}$ as $c / r_{H}$. At the present time:

$$
v=H_{0} r=\frac{c r}{r_{H}}
$$

Now the Friedmann equations indicate a particular density at which the universe must be flat or Euclidean, with curvature parameter $k$ equal to zero. In the absence of a cosmological constant $\Lambda$, the first Friedmann equation becomes: 


$$
H^{2}=\left(\frac{\dot{R}}{R}\right)^{2}=\frac{8 \pi G}{3} \rho-\frac{k c^{2}}{R^{2}}
$$

The critical density for zero curvature at the present time is then given by

$$
\rho_{\text {crit }}=\frac{3 H_{0}^{2}}{8 \pi G}
$$

The universe's evolution and ultimate fate depends on the density parameter, $\Omega=\Omega / \Omega_{\text {crit }}$ which is the ratio of the actual density $\rho$ to the critical value, $\rho / \rho_{\text {crit }}$. The value $\Omega=1$ is specially significant, as the one required for a flat or Euclidean universe, and as the one that separates the closed universe with $\Omega>1$ and spherical geometry, and the open universe with $\Omega<$ 1 and hyperbolic geometry.

Experimentally, since 2000 [4], the value of $\Omega$ has been converging towards the exact value of 1 . This has been interpreted as indicating that the universe is flat and probably infinite, seemingly in line with an inflationary view of cosmology. If this is true, then the general relativistic calculations will be taken at the Newtonian limit, the curvature terms will disappear, and the calculations will yield exactly the same results as Newtonian gravity, with energy incorporated into the mass term. Equation (3) becomes equivalent to $\Omega_{\text {crit }}=3 v^{2} / 8 \pi \rho \mathrm{G} r^{2}$, which is essentially the Newtonian relation $G m / r=1 / 2 v^{2}$, where $m$ is the total mass incorporated within radius $r$.

With the Friedmann equation written as in equation (2), we can easily replace $\rho$ by $\rho+3 P / c^{2}$, to include a vacuum energy term with either positive (inward) or negative (outward) pressure $P$. A positive pressure term, such as would result, for example, from radiation, would make no significant change to the meaning of $\Omega$. However, if the pressure term is negative, it would act as a 'cosmological constant' $\Lambda$, as in following equation:

$$
H^{2}=\left(\frac{\dot{R}}{R}\right)^{2}=\frac{8 \pi G}{3} \rho-\frac{k c^{2}}{R^{2}}-\frac{\Lambda c^{2}}{3}
$$

Seemingly, such a term, the so-called 'dark energy', is now manifested in an outward acceleration in the red-shift velocity of distant galaxies $[1,2]$. If we incorporate $\Lambda$ or the negative pressure term into the density term, we find that it accounts for a high percentage of the total energy of the universe. Original estimates set this at values ranging from 71.4 to 74 per cent with no obvious clue as to its origin, but the most recent data is compatible with another possibility that just as $\Omega$ itself has a critical value 1, which suggests a flat Euclidean universe, so $\Omega_{\Lambda}$, the fraction of $\Omega$ which is due to the dark energy, also has a critical value which would suggest a particular physical explanation for this phenomenon.

\section{A Critical Value}

The data of particular interest comes from the Planck probe. Planck Collaboration XVI, gives 0.6825 as the best fit for $\Omega_{\Lambda}$, and $0.686 \pm 0.020$ for the $68 \%$ confidence limits [5]. With lensing included as well as Planck we obtain 0.6964 as best fit and $0.693 \pm 0.019$ for the $68 \%$ confidence limits. Combining WMap with Planck gives us 0.6817 as best fit and 0.685 +0.018 and -0.016 for the $68 \%$ confidence limits. Planck Collaboration I's overview states that a dark energy contribution determined from temperature anisotropies data alone would give $0.67+0.027$ and -0.023 for the $68 \%$ confidence limits [6].

This experimental value for the dark energy is very close to two-thirds of the total energy of the universe, and this we can show would be a particularly meaningful value for the parameter, with extremely interesting consequences. If we suppose that

$$
\frac{\rho_{v a c}}{\rho_{\text {crit }}}=\frac{2}{3}
$$

then equation (3) gives us a vacuum density of the form

$$
\rho_{v a c}=\frac{H_{0}^{2}}{4 \pi G}
$$

This is equivalent to a negative pressure or 'dark' energy density given by

$$
-P=\frac{H_{0}^{2} c^{2}}{4 \pi G}
$$

and cosmological constant

$$
\Lambda=8 \pi G \rho_{v a c}=2 H_{0}^{2}
$$

Taking $k=0$, and incorporating $P$ (or $\Lambda$ ) into equation (4), we obtain:

$$
H^{2}=\left(\frac{\dot{R}}{R}\right)^{2}=\frac{8 \pi G}{3}\left(\rho+\frac{3 P}{c^{2}}\right)
$$

Putting it into Friedmann's second (acceleration) equation gives:

$$
\frac{\ddot{R}}{R}=\frac{4 \pi G}{3}\left(\rho+\frac{3 P}{c^{2}}\right)
$$

Since, for a flat universe, gravitational action is at the Newtonian limit, there is a clear connection between these equations and an equivalent Poisson equation:

$$
\nabla^{2} \phi=4 \pi G\left(\rho+\frac{3 P}{c^{2}}\right)=4 \pi G\left(\rho-\frac{3 H_{0}^{2}}{4 \pi G}\right)=4 \pi G\left(\rho-3 \rho_{v a c}\right)
$$

If we now assume a uniform and isotropic Hubble universe with mass $m=4 \pi G \rho r^{3} / 3$ and density $\rho$ within radius $r$, then we can express equation (10) in terms of a force on a unit mass, which combines the effect of gravity and dark energy: 


$$
F=\frac{G m}{r^{2}}-H_{0}^{2} r=\left(\frac{4}{3} \pi G \rho-H_{0}^{2}\right) r
$$

That is, the acceleration responsible for dark energy can be expressed as

$$
a=\frac{v^{2}}{r_{H}}=H_{0}^{2} r
$$

A result like this would have special significance, for now the acceleration observed in the red-shift, like the velocity, depends only on Hubble's constant $H_{0}$ and the distance. And it could very well indicate that the dark energy is actually an integral component of the same process that produces the red-shift velocity $v$. Perhaps in fact, it is its own cause, for integrating

$$
a=v \frac{d v}{d r}=H_{0}^{2} r
$$

with respect to $v$ and $r$ between the limits 0 and $v$, and 0 and $r$ gives the exact Hubble red-shift law:

$$
v=H_{0} r=\frac{c r}{r_{H}}
$$

Now equation (14) has been obtained solely from the data, with no conjectural or speculative element whatsoever, and no additional theoretical content or modeling. No assumptions have been made about cosmology. No conclusions have been drawn either, except to add the extra constraint that any possible cosmological theory must accept this velocity-acceleration connection. The equation has in fact, been derived using only the equations of gravitational theory, and that theory can be either general relativistic or Newtonian.

With the data as it is today equation (14) is valid to within the experimental confidence limits, and certainly to within a factor $1.02 \pm 0.02$. The fit is so close that it would be remarkable if $\Omega_{\Lambda}$ should be so close to this critical value without actually reaching it. Certainly, it is of such exceptional physical significance if it is true, that we would be justified in saying that the test of its exact validity should be one of the main aims of future probes. By comparison, we may recall that the Boomerang collaboration claimed with complete confidence in 2000 that finding the $95 \%$ confidence interval for $\Omega$ to be between 0.88 and 1.12 provided 'evidence for a Euclidean geometry of the Universe' [4]. Even on current data, $\Omega=1$ is not established to better than about $1 \%$, tending towards an increased value of $\Omega$ of around 1.01, and inferentially, an increased value of $\Omega_{\Lambda}$. Planck Collaboration I, which includes data from lensing, constrains 'departures from spatial flatness at the percent level', that is $\Omega_{k}=-0.0096$ with $68 \%$ confidence limits of $+0.010-0.0082$, that is, a total $\Omega$ of $1.0096+0.0082-0.010[6]$.

\section{Mach's Principle}

The particular physical significance of equation (14) would seem to be the fact that the velocity term can be derived directly from the acceleration, and vice versa, suggesting that the acceleration, whatever its origin, is actually responsible for the velocity. Though no particular cosmological model is implied by this fact alone, none would be valid that excluded this condition, if we assume that $\Omega_{\Lambda}$ really does equal $2 / 3$.

Yet another equation can be derived directly from (5) and (14), again without any hypothetical or model-dependent input, and this leads to another possible physical interpretation of the dark energy. We start with the acceleration in the form:

$$
a=\frac{d v}{d t}=\frac{c^{2} r}{r_{H}^{2}}
$$

Following this, we recall Sciama's proposal that inertia could be explained along the lines of Mach's principle using a general relativistic gravitomagnetic inductive force between two masses with relative acceleration [7, 8]. In this interpretation, there is an inductive force between any two masses $m_{1}$ and $m_{2}$,

$$
F=\frac{G}{c^{2} r} m_{1} m_{2} \sin \theta \frac{d v}{d t}
$$

which is of the same kind as the one between charges $e_{1}$ and $e_{2}$,

$$
F=\frac{G}{c^{2} r} e_{1} e_{2} \sin \theta \frac{d v}{d t}
$$

which can be derived from Faraday's law of induction. The development of gravitomagnetic equations is essentially a consequence of general relativity, and is one of the standard ways of deriving gravitational waves, but it does not require the complete version of general relativity, and can be relatively easily derived from a special relativistic extension of Newtonian gravitational theory $[9,10]$, in particular using the effects of time dilation and length contraction.

Sciama considered that, using the gravitomagnetic inductive force derived from this theory, and assuming that isotropy removes the angular dependence $\theta$, the inertia of a body of mass $m=m_{1}$ could be attributed to the action of the total mass $m_{H}=m_{2}$ within the observable universe, specified by the Hubble radius $r_{H}$, thus making the inductive force equation equivalent to the Newtonian inertial equation $F=$ $K m a$, with $K$ a constant and $a=d v / d t$. This allows the calculation of the inertial force on a unit mass due to the entire mass in the Hubble universe $m_{H}$ as:

$$
F=\frac{G}{c^{2} r} m_{H} \frac{d v}{d t}
$$

Now, inertia and gravity provide two different standards for defining a unit mass. The connection is normally made via the equivalence principle, and we can apply this here by 
supposing that the radial inertial field of constant magnitude from the centre of a local coordinate system, defined by mass $m_{H}$, equates in magnitude to the gravitational field, $G m_{H} / r_{H}^{2}$, which independently of the local coordinate system, defines a unit of gravitational mass within the same event horizon. This takes the argument beyond Sciama's calculation of inertia, for we then obtain:

$$
\frac{G m_{H}}{c^{2} r} \frac{d v}{d t}=\frac{G m_{H}}{r_{H}^{2}}
$$

Remarkably, this is the exact expression for the acceleration which would result if the dark energy constitutes exactly two-thirds of the total energy of the universe:

$$
a=\frac{c^{2} r}{r_{H}^{2}}=H_{0}^{2} r
$$

Equation (20) is effectively the same as equation (16) rearranged, and with both sides multiplied by $G m_{H}$. In principle, it does not require the development through equations (17) to (19) for its derivation. These simply provide a possible context, which implies that an exact value of two thirds for the dark energy contribution not only links the dark energy and the Hubble red-shift as aspects of the same phenomenon, but may also be of additional interest in connection with Mach's principle and the origin of inertia.

\section{The Relativistic Connection}

If equation (19) is a valid deduction of the inertial force on a unit mass due to the entire mass within the Hubble radius, then the total force will be determined by a combination of the attractive gravitational and repulsive inertial components:

$$
F=-\left(\frac{G m_{H}}{r^{2}}-\frac{G m_{H}}{c^{2} r} \frac{d v}{d t}\right)
$$

which, for a red-shift velocity $v$, can be written

$$
F=-\left(\frac{G m_{H}}{r^{2}}-\frac{G m_{H}}{c^{2} r^{2}} v^{2}\right)
$$

The forms of these equations are interesting for a number of reasons. One is that the inertial acceleration $(d v / d t)$ has exactly the form of an 'inertial force' acceleration $\left(v^{2} / r\right)$, and specifically the fictitious 'centrifugal' outward effect. Its overall positive sign is an indication that this kind of effect is repulsive in opposition to the attractive nature of the force between sources of the same sign. (This could be taken as analogous to the reverse effect in electromagnetism, where the static attraction is repulsive between sources of the same sign, but the magnetic or inductive force between such sources is attractive). Yet another is that the gravitational-inertial combination appears as a Newtonian gravitational force, with a (special) relativistic-type correction. The force equation becomes

$$
F=-\frac{G m_{H}}{r^{2}}\left(1-\frac{v^{2}}{c^{2}}\right)
$$

and the equivalent expression for potential takes the form:

$$
V=-\frac{G m_{H}}{r}\left(1-\frac{v^{2}}{c^{2}}\right)
$$

This is interestingly similar (but different in sign) to the kind of potential function which has been used to generate such things as the general relativistic description of planetary perihelion precession around a body with mass $m$ [11]:

$$
V=-\frac{G m}{r}\left(1+\frac{v^{2}}{c^{2}}\right)
$$

an alternative form of which would be

$$
V=-\frac{G m}{r} /\left(1-\frac{v^{2}}{c^{2}}\right)
$$

In either case, the factor $\left(1-v^{2} / c^{2}\right)$ seems to create something like a reverse effect to the relativistic factors in equations (26) and (27), leading to the flat Euclidean universe which the purely Coulombic static gravitational interaction would demand. This may indicate a deep connection between inertia and gravity which involves the question of the intrinsic speed of the gravitational force.

There has been much confusion over the years as to the meaning of 'the speed of gravity', because many earlier authors naïvely assumed that a relativistic theory necessarily implied speed-of-light transmission. However, it has become increasingly recognized that the gravitational and electrostatic fields are transmitted instantaneously to an infinite distance, moving instantaneously with the source, and do not contain a time-delayed component. This is evident, even from their force laws, which nowhere include the velocity of light. Experimental testing also seems to confirm that this is true for the electrostatic case [12]. The 'speed of gravity' is now taken to mean the speed of gravitational waves and their real carrier boson equivalents, and not that at which the field or static force effect is transmitted. Static 'influences' or 'Coulombic interactions' are always transmitted instantaneously. So, even in a relativistic theory, the mass and charge of a black hole, the sources of the static gravitational and electric fields, are accessible while the finite-velocity radiation generated is suppressed.

Time-delayed information is always associated with localization that is the creation of discrete sources. Now, this happens naturally with the electric field, where the sources are necessarily discrete charges. In the gravitational case, however, the indications point to a very different structure. Gravity is the product of distributed, rather than discrete sources; it arises from the elements of a continuous distribution and has all the characteristics of a vacuum or nonlocal effect rather than a 
local one, especially in regard to the negative energy, which occurs where positive energy is found with charges. Localization occurs because we observe and measure using discrete sources which are associated with charges of some kind (electric, strong or weak) and not because discrete sources are intrinsic to the gravitational field.

The result of this is that the processes of measurement include a localization which is not necessarily intrinsic to gravity but which nevertheless has a very real manifestation. This, we believe, is the true origin of inertia. As its name and its form as a repulsive acceleration equal to $v^{2} / r$ both suggest, it may well be a fictitious effect created by the fact that the localization process is not intrinsic to gravity but is created by our system of measurement. Effectively, gravity is an instantaneous nonlocal correlation, but the process of measurement introduces local time-delayed effects which are equivalent to making an inertial frame appear non-inertial, and so introducing a rotation of the coordinate system which manifests itself as a fictitious force.

\section{Conclusion}

Recent experimental results from the Planck probe give us the real possibility that the dark energy density parameter may exactly equal $2 / 3$. This would be a very significant result because it would make the acceleration and velocity terms in the red-shift phenomenon so closely related that one could be derived from the other by integration / differentiation, and would further imply that they were aspects of a single phenomenon. In addition, the relation has a structure that, without extra physical assumptions, leads to a Machian explanation for inertia.

Now, this development would be in itself remarkable, but prior prediction leading to experimental confirmation remains one of the strongest arguments available for any theoretical construction, and, in this case, there is also a prior prediction. Published versions of the acceleration-velocity relation date back more than thirty years to a calculation done in reverse on the basis of a Machian argument, and the explicit connection with a $2 / 3$ value for $\Omega_{\Lambda}$ was made when experimental results looked less favorable to its conclusions [10, 13]. The calculations of the red-shift acceleration as $H_{0}{ }^{2} r$ were made on the basis of a flat universe, and predated both the experimental discovery of the dark energy and of the flat universe. The most accessible, though not the earliest version of $a=H_{0}{ }^{2} r$, from a series of publications beginning in 1979, was incorporated into a book with a largely historical slant [13].

The potentially critical value for $\Omega_{\Lambda}$ is now within the limits of the data provided by the Planck probe. Future experimental findings could certainly converge on this value, in the same way as they have converged on the physically significant value of unity for $\Omega$. Even a value which came very close would need explanation in the same way as values of $\Omega$ close to 1 were thought to be too close for coincidence even when observations were only within $12 \%$ of the exact value. With the existing Planck data, it would be possible to investigate how the constraints on other cosmological parameters would be affected by applying an exact value of two thirds for the dark energy density, and how any possible deviations in the assumed universal isotropy and uniformity might lead to deviations from the ideal value.

A universe in which the dark energy acceleration was precisely equal to $H_{0}{ }^{2} r$ would be one in which possible cosmologies would be constrained by fundamental laws of physics. Such an exact relation would allow the previously unexpected possibility that fundamental physics would be determining possible cosmologies, rather than the more popular scenario of cosmology determining possible physics. A Machian origin of inertia, for example, would allow a universe to evolve by creating inertial mass at the same time as its space-time structure, with the creation process also generating the force driving its evolution. We can immediately see how such possibilities can be put to rigorous testing using data from future probes.

\section{References}

[1] B. P. Schmidt, et al., vol. 507, ApJ, 1998, p. 46.

[2] S. Perlmutter, et al., vol. 517, ApJ, 1999, p. 565.

[3] A. Friedmann, vol. 10, Z. Phys.,1922, p. 377.

[4] P. de Bernardis, et al., vol. 405, Nature, 2000, pp. 955-959.

[5] Planck Collaboration XVI 2013, arxiv: 1303.5076.

[6] Planck Collaboration I 2013, arxiv:1303.5062.

[7] D. W. Sciama, vol. 113, MNRAS, 1953, p. 34.

[8] D. W. Sciama, The Physical Foundations of General Relativity, New York: Doubleday, 1972.

[9] H. Kolbentsvedt, vol. 56, Amer. J. Phys., 1988, pp. 523-524.

[10] P. Rowlands, Zero to Infinity The Foundations of Physics, Singapore, London and Hackensack, New Jersey: World Scientific, 2007.

[11] A. Harvey, vol. 46, Amer. J. Phys., 1978, pp. 928-929.

[12] A. Calcaterra, R. de Sangro, G. Finocchiaro, P. Patteri, M. Piccolo and G. Pizzella, Measuring Propagation Speed of Coulomb Fields, arXiv:1211.2913, 2012.

[13] P. Rowlands, A Revolution Too Far, Liverpool: PD Publications, 1994. 